REVUE DE L'INSTITUT

FRANÇAIS D'HISTOIRE

EN ALLEMAGNE

\section{Revue de l'IFHA}

Revue de l'Institut français d'histoire en Allemagne

$3 \mid 2011$

IFHA 3

\title{
« Nationenbildung im Museum »
}

Débat d'idées

\section{Marion Deschamp, Stefanie Kohorst et Céline Lebret}

\section{(2) OpenEdition}

Journals

Édition électronique

URL : http://journals.openedition.org/ifha/137

DOI : $10.4000 /$ ifha. 137

ISSN : 2198-8943

Éditeur

IFRA - Institut franco-allemand (sciences historiques et sociales)

Édition imprimée

Date de publication : 6 février 2011

Pagination : 75-78

ISSN : 2190-0078

Référence électronique

Marion Deschamp, Stefanie Kohorst et Céline Lebret, « « Nationenbildung im Museum » », Revue de I'IFHA [En ligne], 3 | 2011, mis en ligne le 01 octobre 2012, consulté le 25 avril 2019. URL : http:// journals.openedition.org/ifha/137; DOI : 10.4000/ifha.137

Ce document a été généré automatiquement le 25 avril 2019

(CIFHA 


\title{
« Nationenbildung im Museum »
}

Débat d'idées

\author{
Marion Deschamp, Stefanie Kohorst et Céline Lebret
}

\section{NOTE DE L'ÉDITEUR}

Francfort-sur-le-Main, 22 juin 2011

L'annonce de la création d'une Maison d'histoire de France ayant soulevé de vifs débats entre historiens français, il a semblé intéressant à l'IFHA de replacer cette question dans une perspective comparative franco-allemande, d'autant plus que les concepteurs du projet français s'appuient explicitement sur l'exemple du Deutsches Historisches Museum (DHM) de Berlin. L'IFHA a donc organisé, en collaboration avec le Forschungszentrum für historische Geisteswissenschaften de l'université Goethe de Francfort, une conférence sur le rôle des musées d'histoire nationale. La discussion réunissait l'historien français Étienne François, membre du comité d'orientation scientifique de la future Maison d'histoire de France, le directeur du musée Historique de Francfort Jan GERCHOW, et l'historienne et commissaire d'exposition Marie-Louise VON PLESSEN. La participation de cette dernière, coconceptrice du musée de l'Europe à Bruxelles, a permis d'élargir la réflexion à une perspective européenne. Le débat était animé par Bernhard JUSSEN (université Goethe de Francfort).

J. GERCHOW, qui a pris la parole en premier, a tenu d'entrée de jeu à endosser le rôle d'«agent provocateur", remettant en cause la pertinence et la légitimité actuelle des musées d'histoire nationale, qu'il interprète comme un phénomène de compensation face à l'effacement de la nation. Si, en effet, la création des musées d'histoire au XIXe siècle joua un rôle dans la construction du sentiment national, leur permanence se révèle désormais, selon lui, rétrograde et à contre-courant de l'affaiblissement de l'idée nationale depuis la fin du XXe siècle. Alors que l'on assiste à la diffraction de la nation comme cadre culturel unitaire, les projets de musées d'histoire nationale connaissent ainsi, de manière paradoxale, une recrudescence. Le DHM et le Haus der Geschichte à Bonn 
sont, pour l'Allemagne, deux témoins phares de cette évolution. Or, aux yeux de J. GERCHOW, l'exposition permanente du DHM proposée depuis 2006 constitue un concept muséologique déjà dépassé, dans la mesure où elle met en scène une histoire nationale linéaire et téléologique, présentant un métarécit chronologique où les changements d'échelles, spatiale et temporelle, ne trouvent pas assez leur place, et d'où sont exclus des thèmes transversaux importants comme l'histoire de l'immigration, ou des approches innovantes comme celle de l'histoire globale. Pour lui, la Maison d'histoire de France ne pourra que souffrir des mêmes travers, dans la mesure où elle ne semble pas complètement indépendante d'intérêts politiques. De plus, elle pourrait arguer de l'existence de la Cité nationale de l'histoire de l'immigration (CNHI) pour occulter du "grand récit national» le phénomène migratoire, et plus largement la relation de la nation à d'autres entités extérieures.

Prenant ensuite la parole, Étienne FRANçoIs a d'emblée exprimé son désaccord avec l'intervention précédente, défendant à la fois le bénéfice du DHM et le projet de la future Maison d'histoire de France, deux dossiers qu'il connait bien pour être membre du conseil scientifique du premier, et membre du comité d'orientation scientifique du second. Rappelant les controverses qui avaient accompagné, à ses débuts, l'exposition du DHM, il a estimé que celle-ci avait finalement tenu ses promesses, à la fois en termes de lisibilité historique et de succès auprès du public. Pour É. FRANçoIs en outre, la mise en question de la nation - comme cadre explicatif - suffisant fait partie intégrante des deux projets muséaux français et allemand et ne justifie pas les violentes critiques de conception rétrograde et nationaliste émises par de nombreux acteurs (historiens, politiciens, personnes civiles) à l'encontre de ces projets. Selon lui, d'ailleurs, les manifestations conflictuelles qui entourent cette «idée ", pour les uns, et "réalité » pour les autres, méritent d'être interrogées et non occultées. Il assure que le projet dirigé par le comité d'orientation scientifique de la future institution française ne présentera pas une vision étriquée de l'identité nationale, selon un mode de définition ontologique, mais prendra en compte l'ensemble des éléments contradictoires et des interprétations plurielles qui y sont attachées.

Après avoir défendu le principe même de musée d'histoire nationale, et ses émanations allemandes comme françaises, É. FRANçoIs a laissé la parole à Marie-Louise VON PLESSEN, favorable quant à elle non à l'abandon de l'échelon national, mais à son traitement dans une dimension européenne. Une institution comme le musée de l'Europe, dont elle est une collaboratrice active, devrait justement jouer un rôle moteur d'intégration, en provoquant un sentiment d'identité européenne, venant s'ajouter et non se substituer à l'identité nationale. M.-L. vON PLESSEN s'est donc prononcée pour un renforcement des projets muséaux au niveau européen, qui s'appuieraient cependant sur le réseau des musées d'histoire nationale pour mettre en valeur la richesse culturelle de l'Europe. Or, la collaboration entre les différents niveaux (national, européen) n'est pour l'heure, estimet-elle, pas assez développée. En fait, la volonté politique des États d'investir dans des musées d'histoire nationale se fait trop souvent aux dépens de l'Europe et de son musée, qui peine à trouver les subsides nécessaires pour faire voir et faire croire, à Bruxelles et ailleurs (selon le principe des expositions itinérantes), à une histoire commune transnationale.

Après avoir chacun exprimé leur point de vue, les trois conférenciers ont été invités à répondre aux questions du public, dont les interventions se sont largement focalisées sur la légitimité (ou non) des musées d'histoire nationale. Pour J. GERCHOW, les musées locaux 
et régionaux sont in fine des lieux plus idoines pour interroger des « identités vécues » et multiplier les représentations de l'histoire. Il estime en effet que la transmission de la mémoire collective s'effectue d'abord et principalement au niveau local. Dès lors, chercher à représenter une identité nationale, dans un lieu unique, comme veut le faire la Maison d'histoire de France, ne lui semble guère approprié. Aux yeux d'É. FRANçoIs, pourtant, la nation reste le cadre de l'exercice de la démocratie. C'est pourquoi la création d'un lieu central qui interroge le concept de nation et ses différentes perceptions lui semble pertinente. La Maison d'histoire de France pourrait, selon lui, refléter les identités locales puisqu'elle serait en dialogue constant avec les mille musées d'histoire locale et régionale répartis sur tout le territoire.

Les relations de la future Maison d'histoire de France avec la CNHI ont fait également l'objet d'une discussion entre É. FRANÇOIS et J. GERCHOW. Pour ce dernier, l'existence de deux institutions séparées est problématique; l'histoire de l'immigration serait en effet exclue de la grande histoire. É. FRANçoIs a certifié qu'une collaboration entre les deux musées était prévue et qu'une histoire décentralisée serait représentée, notamment grâce à la présence de Benjamin Stora (spécialiste de la guerre d'Algérie) au sein du conseil d'orientation scientifique.

Enfin, au vu de ce fourmillement d'implantations muséales différentes, un intervenant du public en est venu à poser la question de l'obsolescence de lieux d'exposition à l'ère du numérique. M.-L. vON PLESSEN a répondu par la nécessité de préserver des lieux réels et de présenter des objets originaux, car on ne saurait, selon elle, renoncer à ce contact direct avec les objets de collection, porteurs d'émotion et enrichissant le parcours visuel du visiteur.

Au terme de la discussion, il est donc apparu que les points de vue variés émis par les intervenants sur la nation, considérée soit en tant que réalité politique et socioculturelle, soit en tant qu'objet d'exposition, dépassaient le cadre étroit d'une discussion entre historiens spécialisés et faisaient sans doute appel, si ce n'est au sentiment national, du moins au sentiment citoyen de chacun, en France comme en Allemagne. 